\title{
An adaptive algorithm for speech analysis and synthesis
}

\author{
Zhongxuan Yuan, Boling Xu, Chongzhi Yu, and Gonghuan Du \\ Institute of Acoustics, State Key Laboratory of Modern Acoustics, Nanjing University, \\ Nanjing 210093, P. R. China
}

(Received 7 December 1996)

\begin{abstract}
In this paper, an artificial neural net architecture for adaptive speech analysis and synthesis is proposed. Based on discussing the signal analysis procedure with mathematical method, the simulation experiment is performed firstly on a typical signal (a square wave). Compared with Fast Fourier Transform, some important characteristics of the Neural Net Signal Analyzer-Synthesizer (NNSAS) are drawn from the experiment results. Then, NNSAS is used for speech signal processing, which results in a new approach to speech analysis and synthesis. In terms of the auditory masking effect, only some major components of connection strength vector are used to produce synthetic speech. Under the high compressive rate conditions, NNSAS can still produce synthetic speech with high articulation.
\end{abstract}

Keywords: Artificial neural net, Speech analysis and synthesis, Fast Fourier transform, Auditory masking effect

PACS number: 43. 72. Ja, 43. 72. Ar

\section{INTRODUCTION}

Since the Fourier Transform was proposed by $\mathrm{J}$. B. J. Fourier in 1822 , the theory of signal analysis has been developing and enriching. Especially, after the Fast Fourier Transform (FFT) algorithm was proposed in 1965, the Discrete Fourier Transform (DFT) has been playing an important role in the field of signal processing. In order to meet the needs of various practical applications, many other orthogonal signal analysis algorithms, such as Discrete Cosine Transform (DCT), Discrete Hartley Transform (DHT), Walsh Transform and Wavelet Transform which is considered to be the breakthrough of Fourier Transform, were proposed.

On the other hand, it was discovered that some biological nervous systems also have the capability of signal analysis, such as mammal's auditory systems. ${ }^{1-3)}$ In 1960, G. V. Békésy4) presented his famous discovery of the phenomenon of constant wave and local consonance of basilar membrane, which showed the signal analysis characteristics of auditory nerve. The phenomenon prompts us to explore artificial neural net to imitate the signal analysis functions in biological nervous systems.

Artificial Neural Net (ANN) models are used to attempt to achieve real-time response and humanlike performance using numerous simple processing units operating in parallel as in biological nervous systems. These models have the greatest potential in fields such as speech and image signal processing where many hypotheses are pursued in parallel, high computation rates are required, and the performance of the current best system is far below that of human. In recent years, ANN models are widely used to perform many specific tasks. Compared with their applications to other research subjects, ANNs are less used in signal analysis and synthesis. But we can still find some contributions to the subject. For example, A. D. Culhane et al., ${ }^{5)}$ used the linear programming neural net, which was proposed by Tank and Hopfield, $\left.{ }^{6}\right)$ to compute the Discrete Hartley and Discrete Fourier Transforms.

In this paper, a neural net model, which is used to 
implement an adaptive algorithm for speech analysis and synthesis, is proposed. We call it Neural Net Signal Analyzer-Synthesizer (NNSAS). The purpose of this work is to explore a new approach to speech analysis and synthesis, which processes signals in the way similar to that in biological neural systems. The goal is to get better representations of speech signals (such as extracting speech features for speech synthesis or for speech recognition). The paper is organized as follows. In section 2, a fundamental topology of a neural net is given for showing the possibility of signal analysis with NNSAS based on mathematical analysis method. Special attention is drawn onto the specific design choices concerning the number of analysis functions. A complete configuration is proposed and its adaptive analysis process and algorithm are discussed in section 3. Computer simulation experiments are performed in section 4. Some merits of NNSAS are indicated and compared with that of FFT. In section 5, NNSAS is used as speech analyzer and synthesizer. At last, we will summarize and conclude this paper in section 6 .

\section{THE POSSIBILITY OF SIGNAL ANALYSIS WITH ANN}

The conventional signal analysis is based on a set of linearly orthogonal functions. A composite signal can be represented by the linear combination of the set of functions. The purpose of signal analysis is to make the obscure features in original signal clearer or to fine better representation of original signal. Supposed that there is a continuous-time signal $y(t)$ and a set of orthogonal functions $x_{1}(t)$, $x_{2}(t), \cdots, x_{M}(t)$ which are formed of the $M$ dimensional orthogonal signal space. If the signal space is complete in variable region $\left(t_{1}, t_{2}\right)$, signal $y(t)$ which conforms to Dirichlet conditions could be represented with the linear combination of the $M$ functions

$$
y(t)=\sum_{m=1}^{M} c_{m} x_{m}(t)
$$

In order to meet the need of different signal analysis, various kinds of orthogonal functions $x_{m}(t), 1 \leq m$ $\leq M$, are proposed, which result in many kinds of linear orthogonal signal analysis algorithms.

Because of the nonlinearity of biological neural nets, it is clear that neural nets perform signal analysis in a different way. To embody the nonlinear

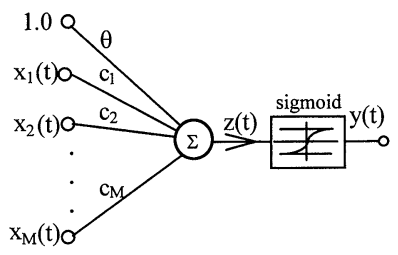

Fig. 1 Fundamental topology of a neural net for signal analysis.

character of the neural nets, Eq. (1) is modified as

$$
y(t)=f\left(x_{1}(t), x_{2}(t), \cdots, x_{m}(t), \cdots\right)
$$

where $f(\cdot)$ denotes the nonlinearity of a neural net. Figure 1 shows a fundamental topology which is called a single-layer perceptron. ${ }^{7)}$ How could the simple topology be used to implement signal analysis?

It is known that a neural net is characterized by an internal offset or threshold $\theta$ and by the type of nonlinearity. In order to perform the task of signal analysis, the input vector, $\vec{X}(t)=\left[x_{1}(t), x_{2}(t), \cdots\right.$, $\left.X_{m}(t)\right]$, which is composed of $M$ components is explained as a set of analysis functions. It is very important to deal with input vector in terms of this idea. Based on this idea, the analysis results are considered to be kept in the connection strength vector $\vec{C}=\left[c_{1}, c_{2}, \cdots, c_{M}\right]^{\mathrm{T}}$ whose functions correspond to that of synapses in a biological neuron. Assuming that the representative nonlinearity is the Sigmoidal function. The topology in Fig. 1 can be used to represent a signal $y(t)$, that is

$$
y(t)=\frac{1}{1+e^{-z(t) / \theta_{0}}}
$$

where

$$
z(t)=c_{1} x_{1}(t)+c_{2} x_{2}(t) \cdots+c_{M} x_{M}(t)+\theta
$$

the relationship between $y(t)$ and $z(t)$ is

$$
z(t)=\theta_{0} \cdot \ln \left(\frac{y(t)}{1-y(t)}\right)
$$

Equation (4) is used to show the possibility of signal analysis with the single-layer perceptron. Now our attention is drawn onto the number of analysis functions to find unique solution to Eq. (4). If variable $t$ in the analysis region $\left(t_{1}, t_{2}\right)$ is divided into $N$ sample points (comply to the uniform sampling theorem) for computer simulation, Eq. (4) can be rewritten as 


$$
\left[\begin{array}{ccccc}
x_{1}\left(t_{1}\right) & x_{2}\left(t_{1}\right) & \cdots & x_{M}\left(t_{1}\right) & 1.0 \\
x_{1}\left(t_{2}\right) & x_{2}\left(t_{2}\right) & \cdots & x_{M}\left(t_{2}\right) & 1.0 \\
\vdots & \vdots & \vdots & \vdots & \vdots \\
x_{1}\left(t_{N}\right) & x_{2}\left(t_{N}\right) & \cdots & x_{M}\left(t_{N}\right) & 1.0
\end{array}\right]\left[\begin{array}{c}
c_{1} \\
c_{2} \\
\vdots \\
c_{M} \\
\theta
\end{array}\right]=\left[\begin{array}{c}
z\left(t_{1}\right) \\
z\left(t_{2}\right) \\
\vdots \\
z\left(t_{N}\right)
\end{array}\right]
$$

Given a signal $y(t)$, the process of signal analysis in the neural net is that of adaptive adjusting the connection vector $\vec{C}$. This process is equivalent to find the solution to Eq. (6). For further discussion, we will ignore the differences between threshold $\theta$ and connection $c_{m}$, and assume that the total number of $c_{m}$ and $\theta$ is $M$. Equation (6) can be simplified as

$$
X \vec{C}=\vec{Z}
$$

where $X$ is a two-dimensional matrix of size $N \times M$.

In order to ensure for an unique solution to Eq. (7), the analysis functions, $x_{1}(t), x_{2}(t), \cdots, x_{M}(t)$, should be orthogonal or linear independence, and $N=M$. If the value of the determinant $X$ is not zero, that is, Det $X \neq 0$, then

$$
\vec{C}=X^{-1} \vec{Z}
$$

If $N<M$, there will exist many solutions to Eq. (7). These solutions are only in keeping with the constrain conditions of small number of samples of original signal. It is clear that these solutions are not expected. We have to reduce the value of $M$ till $N=M$ to ensure an unique solution to Eq. (7).

If $N>M$, although there is a solution, $\vec{C}=$ $\left(X^{\mathrm{T}} X\right)^{-1} X^{\mathrm{T}} \vec{Z}$, to Eq. (7), it is not acceptable because a large error is still existed when the analysis procedure is finished. So, we have to append the number of analysis functions, $M$, till $M=N$.

As discussed above, it is possible to represent signals with ANN. Obviously, Eq. (7) can be solved algebraically without using nonlinear function in Eq. (3) if we let $z(t)=y(t)$. However, this method belongs to linear signal analysis algorithms which are widely explored. Our efforts are made to find a new approach to speech signal processing, which processes signals in the way similar to that in biological nervous systems. For this reason, we introduce the nonlinear function to solve Eq. (7) adaptively with ANN. The detail of the algorithm is described in next section. Its merit and novel points are discussed in sections 4 and 5 and summarized in section 6 .

\section{ARCHITECTURE OF NNSAS AND ADAPTIVE ANALYSIS PROCEDURE}

\subsection{A Complete Architecture for Adaptive Signal Analysis}

It is known that an ANN model which is used to perform specially appointed task must be specified the net topology, nonlinear characteristics, and training or learning algorithm. Therefore, a learning rule must be given to Fig. 1. In this section, the delta rule $^{8)}$ is adapted as the learning algorithm to describe the procedure of adaptive signal analysis in the neural net. Thus, we have a complete architecture which is shown in Fig. 2.

In Fig. 2, a function extending unit is put in the frond-end of a single-layer perceptron shown in Fig. 1. The extending unit is used to extend the variable $t$ with a set of orthogonal functions which are used as analysis functions. We can see the core element of Fig. 2 is the functional-link net which was proposed by Dr. Sobajic D. and Y. H. Pao et $a .^{9-11)}$ Functional-link net was successfully used in speech and speaker recognition systems by us. $^{12,13)}$

\subsection{Analysis Procedure}

Mathematically, the delta $(\delta)$ rule is gradient descent of the mean-squared error as a function of the connection strengths. The adaptive signal analysis procedure performs two passes, forward and backward passes, through NNSAS.

During the forward pass, variable $t$ in region $\left(t_{1}\right.$, $\left.t_{2}\right)$ is expanded into an $M$-dimensional vector $\vec{X}(t)$ with a set of orthogonal functions

$$
\vec{X}(t)=\left[x_{1}(t), x_{2}(t), \cdots, x_{M}(t)\right]
$$

The input of the net is the sum of the components of

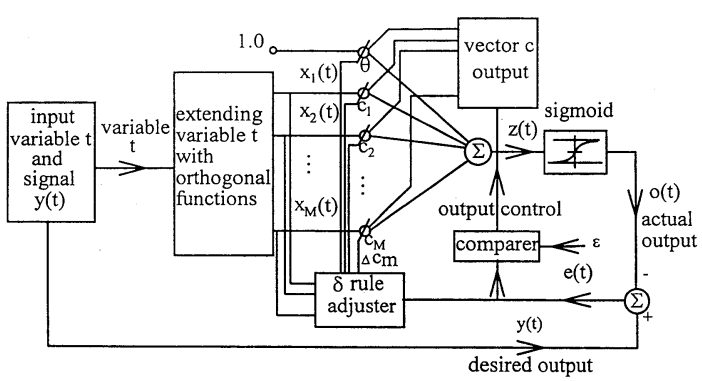

Fig. 2 Block diagram of Neural Net Signal Analyzer-Synthesizer. 
vector $\vec{X}(t)$ and threshold $\theta$, which are firstly weighted with the current connection strengths. The initial strengths and $\theta$ are small random values from -0.5 to +0.5 . Thus

$$
z(t)=\vec{X}(t) \vec{C}+\theta
$$

the actual output of the net can be calculated with the following equation.

$$
o(t)=f(z(t))
$$

where $f(\cdot)$ is the nonlinearity. Among three common types of nonlinearities of neural net (i.e. hard limiter, threshold logic and sigmoid), sigmoid is the most proper one for this occasion because most signals are with continuous value. So,

$$
o(t)=\frac{1}{1+e^{(\mathcal{C} X(t)+\theta) / \theta_{0}}}
$$

where $\theta_{0}$ is used to change the sigmoid nonlinearity. The actual output $o(t)$ is then compared to the desired output $y(t)$, the signal which is being analyzed. Then the error is calculated.

$$
e(t)=\frac{1}{2}(y(t)-o(t))^{2}
$$

During the backward pass, the derivation of this error is then propagated back through NNSAS, and all the connection strengthens are adjusted so as to decrease the error.

$$
\begin{aligned}
\Delta c_{m} & =-\eta \frac{\partial e(t)}{\partial c_{m}} \\
& =\eta \cdot(y(t)-o(t)) \cdot o(t) \cdot(1-o(t)) \cdot x_{m}(t)
\end{aligned}
$$

In order to improve the convergent properties of NNSAS, a momentum term is added to Eq. (14).

$$
\begin{aligned}
& \Delta c_{m}(t+\Delta t) \\
& \begin{aligned}
= & \eta(y(t)-o(t)) \cdot o(t) \cdot(1-o(t)) \cdot x_{m}(t) \\
& +\alpha \cdot \Delta c_{m}(t)
\end{aligned}
\end{aligned}
$$

In Eqs. (14) and (15), the analysis rate (or adaptive step size) $\eta$ and the momentum rate $\alpha$ are small positive scalars that control the transient and steadystate convergent properties of the algorithm. Both $\eta$ and $\alpha$ are determined empirically through experiments.

The forward and backward passes are repeated many times for all sample points of variable $t \in\left(t_{1}\right.$, $t_{2}$ ) till NNSAS converges to produce the desired output $y(t)$ or the system error $E$ is small enough,

$$
E=\frac{1}{t_{2}-t_{1}} \int_{t_{1}}^{t_{2}}(y(t)-o(t))^{2} d t \leq \varepsilon
$$

Under this condition, the projective values of the signal $y(t)$ in each analysis function $x_{m}(t), 1 \leq m \leq$ $M$ are represented by the connection strength $C_{m}$, $1 \leq m \leq M$.

\subsection{Synthesis Procedure}

When NNSAS finishes the analysis process, the connection strength $c_{m}, 1 \leq m \leq M$, could be used to reproduce (synthesize) the synthetic signal $\hat{y}(t)$ according to the forward pass (Eqs. (9)-(12)) in analysis procedure described in subsection 3.2. It is obvious that the synthesis procedure is direct and simple.

\section{COMPARISON EXPERIMENT}

In this section, we first show some experimental results from the Neural Net Signal AnalyzerSynthesizer (NNSAS) performing on square waves. Then the results of FFT (for analysis) and inverse FFT (for synthesis) will be given for showing the differences between NNSAS and FFT.

4.1 Square Wave Analysis and Synthesis with NNSAS

Supposed that the matrix $X$ in Eq. (6) is

$$
\left[\begin{array}{cccccc}
t_{1} & \sin \left(1 \cdot \Omega \cdot t_{1}\right) & \cos \left(1 \cdot \Omega \cdot t_{1}\right) & \cdots & \sin \left(\frac{M}{2} \cdot \Omega \cdot t_{1}\right) & 1.0 \\
t_{2} & \sin \left(1 \cdot \Omega \cdot t_{2}\right) & \cos \left(1 \cdot \Omega \cdot t_{2}\right) & \cdots & \sin \left(\frac{M}{2} \cdot \Omega \cdot t_{2}\right) & 1.0 \\
\vdots & \vdots & \vdots & \vdots & \vdots & \vdots \\
t_{N} & \sin \left(1 \cdot \Omega \cdot t_{N}\right) & \cos \left(1 \cdot \Omega \cdot t_{N}\right) & \cdots & \sin \left(\frac{M}{2} \cdot \Omega \cdot t_{N}\right) & 1.0
\end{array}\right]
$$

where $\Omega=2 \pi / T$. The signal $y(t)$ which is being analyzed is represented in Eq. (17). Figure 3 shows the system error $E$ as a function of the number of adaptation cycles when $\eta=0.15, \alpha=0.62$ and samples on $y(t)$ are $80(N=80)$. This figure indicates that the system has a good convergent characteristic.

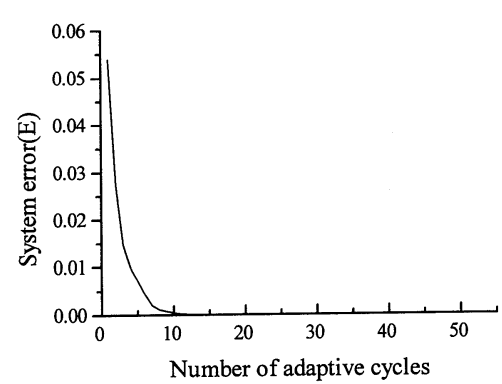

Fig. 3 Convergence characteristics of NNSAS. 


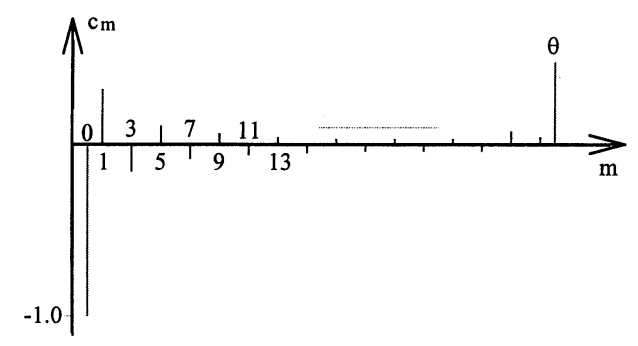

(a) The neural-spectrum of square wave $(N=32)$.

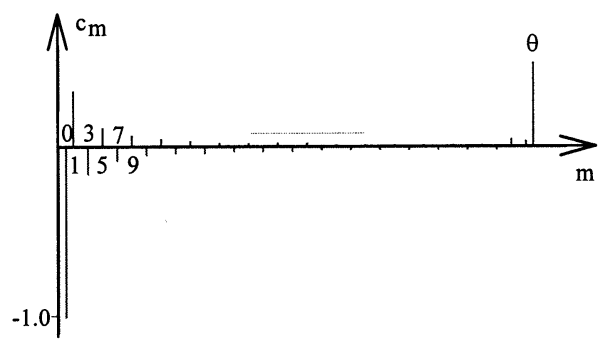

(b) The neural-spectrum of square wave $(N=64)$.

Fig. 4 The neural-spectrums of the square waves analyzed with NNSAS.
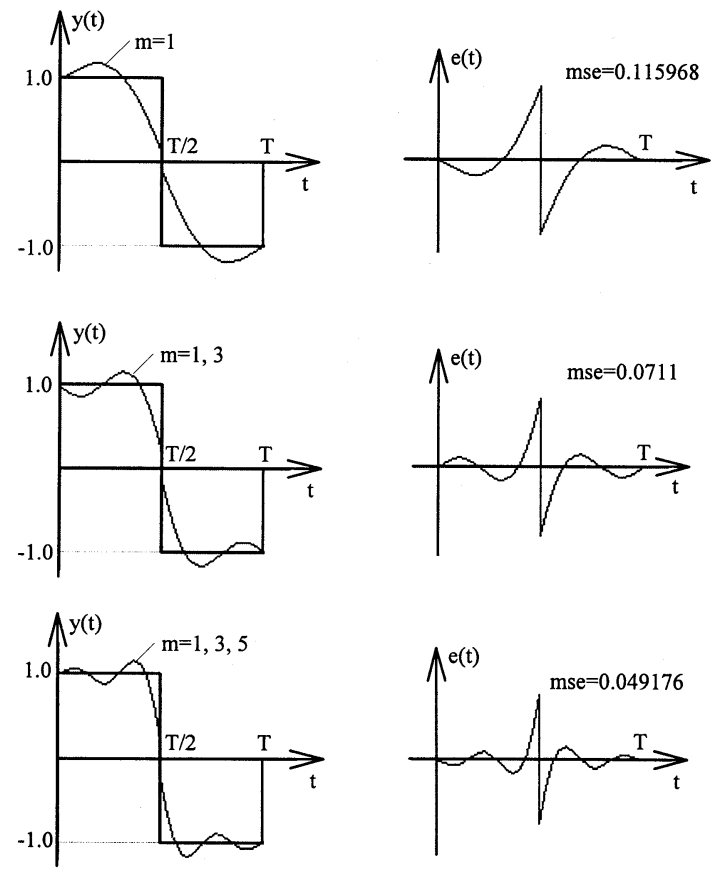

Fig. 5 The original and synthetic square waves (left), the errors (right) between the original and synthetic square waves based on NNSAS. The synthetic waves are produced with partial components of connection strength vector.
The analysis results, the connection strengths, are drawn in Fig. 4. Since the signal analysis is carried out with neural net, the "spectrum" is called neuralspectrum in order to distinguish it from that of FFT.

$$
y(t)= \begin{cases}1 & 0<t<T / 2 \\ -1 & T / 2<t<T\end{cases}
$$

In Fig. 4 (a), the samples on the square wave are 32 $(N=32)$ and in Fig. 4 (b) $N=64$. Because the projective values in COS terms are small enough to be omitted, we can think that the neural-spectrums are valued only in SIN terms. Figure 5 shows the original square wave, synthetic square waves reproduced (synthesized) with partial components of connection strength vector $\vec{C}$ and the differences (errors) between the original and synthetic square waves. The mean squared errors (mse) are printed in right upside corner under each synthetic condition.

4.2 Square Wave Analysis and Synthesis with FFT and IFFT

Discrete Fourier Transform (DFT) has led to the

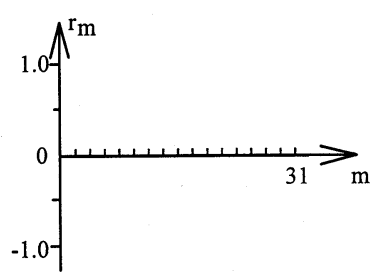

(a) Real part.

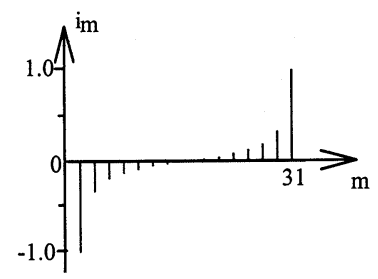

(b) Imaginary part.

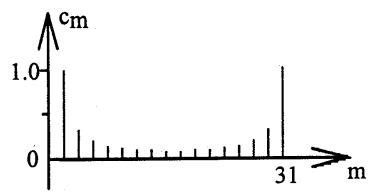

(c) Linear power spectrum.

Fig. 6 Spectrum of square wave analyzed with FFT. 

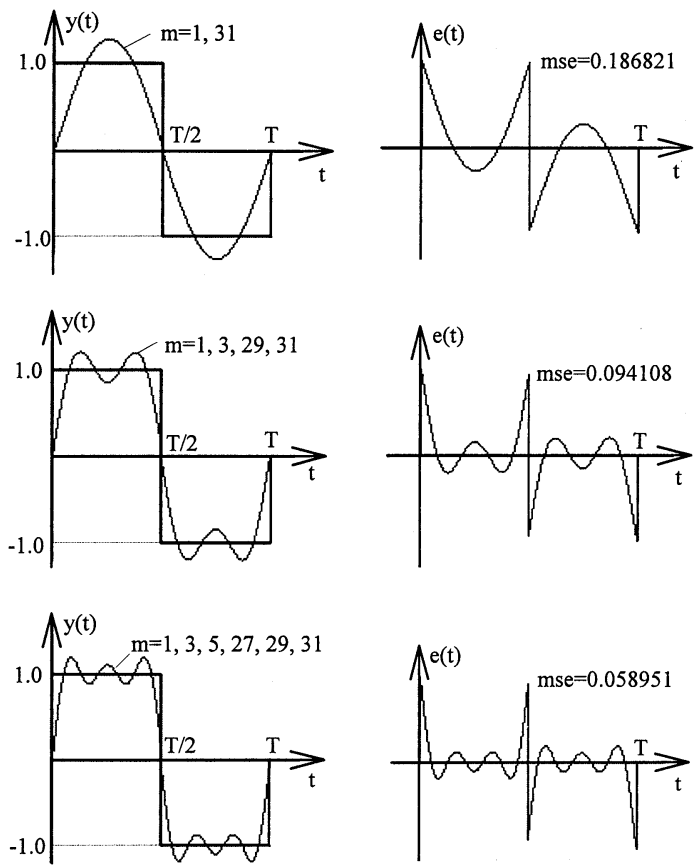

Fig. 7 The original and synthetic square waves (left) and the errors (right) between the original and synthetic square waves (right) with FFT and IFFT. The synthetic waves are produced with partial terms of FFT.

development of one of the most useful tools for speech analysis. Mathematically, DFT is based on linear signal analysis. Some auditory model used DFT as the traditional form of preprocessing. ${ }^{14)}$ In order to facilitate comparison, FFT is used to analyze the same square wave listed in Eq. (17). The FFT spectrum of the square wave is shown in Fig. 6.

Figure 7 shows the original square wave, synthetic square waves synthesized from partial coefficients and the mse (printed in right upside corner) between the original and synthetic square waves.

\subsection{Contrast and Discussion}

When Fig. 4(a) and 4(b) are compared with Fig. 6, we find that neural spectrums are valued very small in COS terms and the values of the real part of FFT (Fig. 6(a)) are also very small. This is because the square wave is an odd function. However, the envelops of the two kinds of spectrums are quite different. From Fig. 6, we can see that $c_{m}$ of FFT has the feature of symmetry, so only half of $c_{m}$ are effective. Thus, FFT has lower frequency reso-

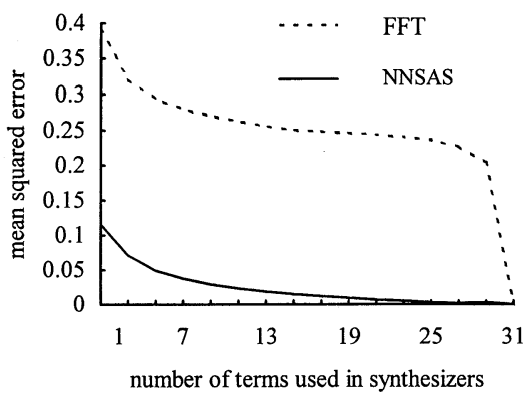

Fig. 8 Mean-squared errors between the original and synthetic square waves versus the synthetic terms. The samples on square waves are 32 .

lution than NNSAS at the same analysis length and sample rate. Because the coefficients of FFT are composed of real parts and imaginary parts, these produce the complicated phase feature which makes much trouble to researchers in homomorphic speech processing. On the other hand, Fig. 5 and Fig. 7 (In order to better show the synthetic wave, the symmetric terms are partially used in couples in inverse FFT) show that the two groups of errors are quite unlike. Figure 8 shows the mean-squared errors between the original and synthetic square waves versus the synthetic terms in the two kinds of synthesizers. It is clear that the mean-squared errors produced by neural net synthesizer are much lower than that produced by Fourier synthesizer in the same synthetic terms.

An important character of NNSAS is that the analysis function can be easily replaced by other kinds of orthogonal functions. An example with this feature is to be given in following section.

\section{EXPERIMENT ON SPEECH ANALYSIS AND SYSTHESIS}

In this section, the Neural Net Signal AnalyzerSynthesizer (NNSAS) is used for speech analysis and synthesis. In order to demonstrate the extensiveness of selecting the analysis functions, the matrix $X$ in Eq. (6) is replaced by

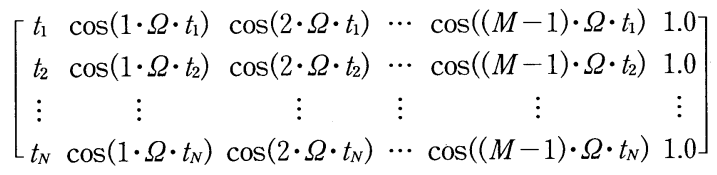

where $\Omega=2 \pi / T$ and $N=80$. That there is no restriction on analytic length $N$ is another interest- 


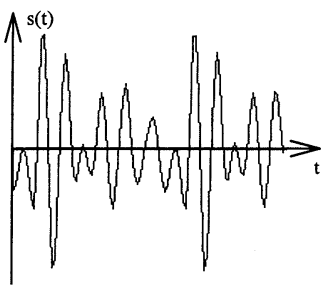

(a) Original wave form.

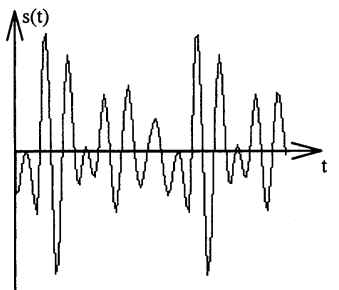

(c) Synthetic wave form $\left(P_{\mathrm{r}}=1\right)$.

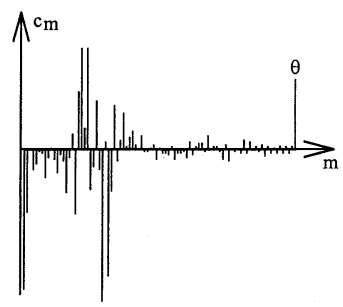

(b) Neural-cosine spectrum.

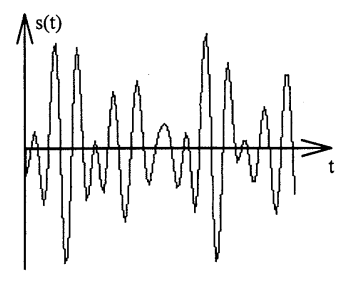

(d) Synthetic wave form $\left(P_{\mathrm{r}}=10\right)$.

Fig. 9 A segment of the original waveform of vowel /a/ and its neural-cosine spectrum as well as synthetic wave forms.

ing feature of NNSAS. Figure 9 (a) is a segment of speech wave, $s(t)$, taken from vowel /a/ in time domain. Its neural-cosine spectrum analyzed by the NNSAS is shown in Fig. 9(b). Figure 9(c) is noncompressive $\left(P_{\mathrm{r}}=1\right)$ synthetic speech waveform, $\hat{s}(t)$. Figure $9(\mathrm{~d})$ is the compressive synthetic speech waveform, $\hat{s}(t)$, when the compressive rate is ten, that is, $P_{\mathrm{r}}=10$. The compressive rate is defined as

$$
P_{\mathrm{r}}=\frac{M}{M_{\mathrm{r}}}
$$

where $M$ is the number of components in connection strength vector $\vec{C}, M_{\mathrm{r}}$, is the number of components taken from vector $\vec{C}$ to reproduce the synthetic waveform. Considering the masking properties of the human ear, ${ }^{15,16)}$ only some components of vector $\vec{C}$, which are formed of major formants, are used in the NNSAS to get the synthetic speech signal. In order to quantitatively express the benefits of NNSAS, the results of segmental Signalsynthetic Noise Ratio $(S N R)$ have been examined.

$$
S N R=10 \log _{10} \frac{E\left[s^{2}(n)\right]}{E\left[e^{2}(n)\right]}=10 \log _{10} \frac{\sigma_{s}^{2}}{\sigma_{e}^{2}}
$$

where

$$
e(n)=s(n)-\hat{s}(n)
$$

Figure 10 shows the segmental $S N R$ versus the

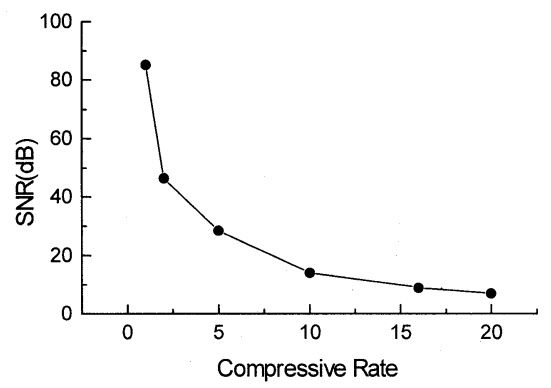

Fig. 10 Segmental SNR versus the compressive rate $P_{\mathrm{r}}$.

compressive rate $P_{\mathrm{r}}$.

Figure 11(a) shows the 12th-order LPC-FFT spectrum of original Chinese voice nanjing (南京). Figure 11(b) and (c) are the 12th-order LPC-FFT spectrums of synthetic voices for $P_{\mathrm{r}}=4$ and $P_{\mathrm{r}}=10$ respectively. When $P_{\mathrm{r}}=10$, the spectrum of synthetic speech is very different from that of original speech, but the major consonants are still kept well. So the synthetic speech is still with high articulation, and the speaker's individual features are also kept well. If a proper encode method is introduced, low bit rate representation for speech signal can be implemented.

\section{SUMMARY AND CONCLUSION}

In this paper, a new approach to adaptive speech analysis and synthesis which is based on artificial neural net was presented. Firstly, we used a fundamental topology of a neural net for explaining the possibility of signal analysis and synthesis with NNSAS in mathematical way. Then a complete architecture for adaptive signal analysis and synthesis was implemented. The analysis and synthesis procedures were also discussed. Comparative simulation experiments were performed on typical signal, square waveform, both with NNSAS and FFT. We discovered that $\mathrm{i}$ ) NNSAS has higher frequency resolution than FFT; ii) there is no complicated phase problem in NNSAS; iii) the analysis functions can be easily replaced by other orthogonal functions; iv) the shapes of envelopes in both spectrums are quite different and some other interesting characteristics of NNSAS were also discussed. When NNSAS was used for speech analysis and synthesis, high articulation synthetic speech voices have been gotten from NNSAS under high compressive rate. The speaker's individual features are also 


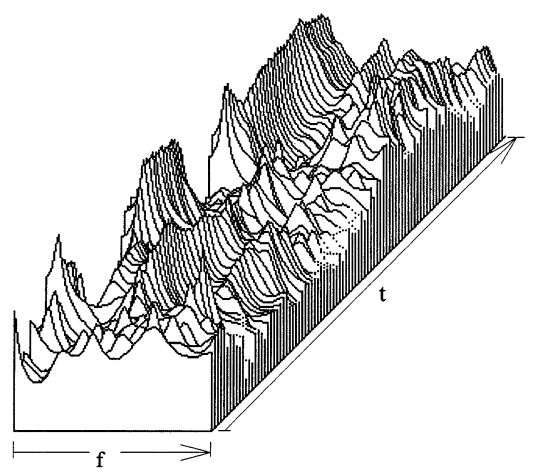

(a) The spectral envelope by LPC-FFT for original speech.

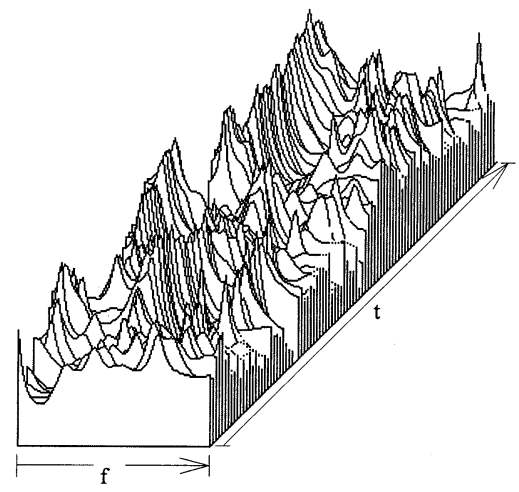

(b) The spectral envelope by LPC-FFT for synthetic speech $\left(P_{\mathrm{r}}=4\right)$.

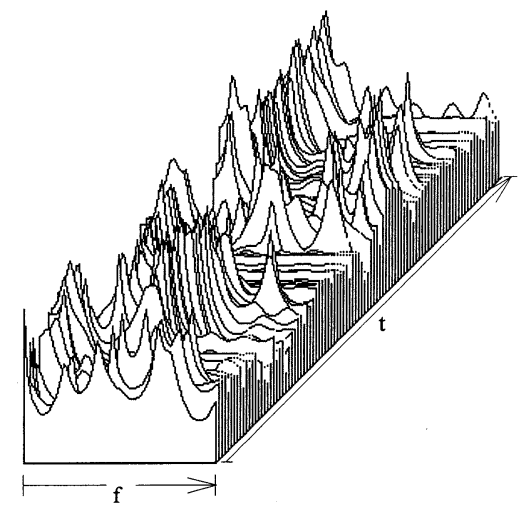

(c) The spectral envelope by LPC-FFT for synthetic speech $\left(P_{\mathrm{r}}=10\right)$.

Fig. 11 The original spectrum and synthetic spectrums of Chinese speech nanjing (南京). The all-pole polynomial-fit is a 12th-order LPC for FFT spectrum. kept well in the synthetic speech voices. The theoretic analysis and experimental results showed that the approach to adaptive signal analysis and synthesis based on artificial neural net is an effective one to signal processing such as speech analysis and synthesis.

\section{ACKNOWLEDGEMENTS}

The authors would like to thank Professor Daochun Yang and Professor Yiman Hua for their useful discussions. The authors would also like to thank the reviewers whose comments and suggestions have improved the quality of this paper. This work was partly supported by the National Science Foundation of China and partly supported by State Key Laboratory of Modern Acoustics at Nanjing University.

\section{REFERENCES}

1) W. Rhode, "Observations of the vibrations of the basilar membrane in squirrel monkey using the Mössbauer technique," J. Acoust. Soc. Am. 49, 12281231 (1971).

2) D. O. Kim, C. Molnar and J. W. Mathews, “ Cochlea mechanics: Nonlinear behavior in two-tone responses and in ear-canal sound pressure," $\mathbf{J}$. Acoust. Soc. Am. 67, 1704-1721 (1980).

3) M. Miller and M. Sachs, "Representation of step consonants in the discharge patterns of auditorynerve fibers," J. Acoust. Soc. Am. 74. 502-517 (1983).

4) G. V. Békésy, Experiments in Hearing (McGrawHill, New York, 1960).

5) A. D. Culhane, M. C. Peckerar, and C. R. K. Marrian, "A neural net approach to discrete Hartley and Fourier transforms," IEEE Trans. CAS 36, 695-703 (1989).

6) D. W. Tank and J. J. Hopfield, "Simple 'neural' optimization networks: An A/D converter, signal decision circuit, and a linear programming circuit," IEEE Trans. CAS 36, 533-541 (1986).

7) R. P. Lippmann, "Neural nets for computing," IEEE Proc. ICASSP 88, 1-6 (1988).

8) D. E. Rumelhart, G. E. Hinton, and R. J. Williams, "Learning internal representation by error propagation," in Parallel Distributed Processing: Explorations in the Microstructures of Cognition, Vol. 1., D. E. Rumelhart and L. McClland, Eds. (MIT Press, Cambridge, MA, 1986), pp. 318-362.

9) D. Sobajic, "Neural nets for control of power systems," Ph. D. Thesis, Computer Science Dept., Case Western Reserve University, Cleveland, OH (1988).

10) Y. H. Pao, Adaptive Pattern Recognition and Neural Networks (Addison-Wesley, New York, 1989).

11) M. S. Klassen and Y. H. Pao, "Characteristics of the functional-link net: A higher order delta rule net," 


\section{Z. YUAN et al. : ADAPTIVE ALGORITHM FOR SPEECH ANALYSIS AND SYNTHESIS}

IEEE Proc. ICNN, June (1988).

12) Zhong-Xuan Yuan, Bo-Ling $\mathrm{Xu}$, and Chong-Zhi $\mathrm{Yu}$, "Speech recognition based on fuzzy-neural network," IEEE Proc. ICNNSP, Vol. I, 808-811, Dec. (1995).

13) Zhong-Xuan Yuan, Bo-Ling Xu, and Chong-Zhi Yu, "A kind of fuzzy-neural networks for textindependent speaker identification," IEEE Proc. ICASSP 96, 657-660, May (1996).

14) K. L. Payton, "Vowel processing by a model of the auditory periphery: A comparison to eighth-nerve reposes," J. Acoust. Soc. Am. 83, 145-162 (1988).

15) M. R. Schroeder, B. S. Atal, and J. L. Hall, "Optimizing digital speech coders by exploiting masking properties of the human ear," J. Acoust. Soc. Am. 66, 1647-1652 (1979).

16) B. Scharf, "Critical bands," in Foundations of Modern Auditory Theory, J. V. Tobias, Ed. (Academic Press, New York, 1970), pp. 159-202. 